Relations industrielles

Industrial Relations

\title{
Chômage et évolution des structures, Bureau International du Travail, Genève, 1962, 245 pp.
}

\section{Jacques St-Laurent}

Volume 18, numéro 2, avril 1963

URI : https://id.erudit.org/iderudit/1021445ar

DOI : https://doi.org/10.7202/1021445ar

Aller au sommaire du numéro

Éditeur(s)

Département des relations industrielles de l'Université Laval

ISSN

0034-379X (imprimé)

1703-8138 (numérique)

Découvrir la revue

Citer ce compte rendu

St-Laurent, J. (1963). Compte rendu de [Chômage et évolution des structures, Bureau International du Travail, Genève, 1962, 245 pp.] Relations industrielles / Industrial Relations, 18(2), 286-287. https://doi.org/10.7202/1021445ar

Tous droits réservés @ C Département des relations industrielles de l’Université Laval, 1963
Ce document est protégé par la loi sur le droit d'auteur. L’utilisation des services d'Érudit (y compris la reproduction) est assujettie à sa politique d'utilisation que vous pouvez consulter en ligne.

https://apropos.erudit.org/fr/usagers/politique-dutilisation/ 
Le professeur Turner a choisi d'étudier ces questions au moyen d'une étude comparée de plusieurs syndicats du textile en Angleterre, non seulement entre ces derniers, mais aussi avec d'autres grandes centrales syndicales de ce pays.

Les syndicats du textile en Angleterre, se prêtent d'autant mieux à une telle étude qu'ils sont parmi les plus représentatifs du trade-unionisme britannique, qu'ils ont l'une des plus longues histoires, et que l'importance de cette industrie et l'expérience syndicale de ces associations sont parmi les plus grandes dans ce pays.

L'auteur se sert d'abord de l'histoire comme point de départ de son étude. A cette fin, il fait l'historique des syndicats du textile depuis leurs débuts en retraçant le développement et la structure des métiers du textile et en exposant brièvement l'état récent du tradeunionisme chez les fileurs.

L'étude se divise en cinq livres comptant chacun deux chapitres. Le premier livre retrace l'organisation primitive des fileurs et l'auteur $y$ suggère que cette organisation a des racines beaucoup plus lointaines et profondes que l'on présuppose généralement, et que sa continuité historique est bien plus réelle qu'on a cru jusqu'ici.

Disons tout de suite ici que ces différentes parties ou elivres», ainsi que les chapitres qui les composent ne tracent pas une ligne historique continue du sujet, mais que cette ligne est entrecoupée de comparaisons entre organisations étudiées et aussi entre les problèmes du passé et ceux de nos jours où l'auteur découvre plusieurs similitudes là où on aurait cru à des phénomènes purement modernes.

Le deuxième livre est consacré, pour sa part, au développement du tradeunionisme actuel dans l'industrie textile surtout quant aux techniques d'action et aux formes institutionnelles avec lesquelles il est présentement identifié. L'auteur dans les deux chapitres qui le composent, substitue à l'interprétation traditionnelle de la lutte des syndiqués et du patron, (qu'il ne considère pas pour autant comme non avenue) celle de la lutte entre les diverses catégories de travailleurs et des cunions» qui les représentent sur le marché du travail, en matière d'organisation, de revendications, de contrôle et de représentation.
Le livre troisième élargit les préoccupations de l'étude en comparant l'évolution des cunions» de fileurs à celle d'autres catégories majeures d'employés, afin de tester les conclusions tirées à l'occasion de l'étude des syndicats du textile.

Et l'auteur continue cette histoire comparé tout au long du quatrième livre en insistant davantage sur l'organisation interne des syndicats étudiés, leur gouverne, les relations leadership-membership, la démocratie syndicale, etc.

Enfin le cinquième livre s'attache plus particulièrement à comparer certaines questions particulières entre les syndicats en général et ceux des fileurs: par exemple, le degré de créponse des syndicats aux pressions sociales environnantes dans l'application de leurs constitutions respectives en regard des droits et privilèges de leurs membres, le degró d'adaptation de leurs structures aux impératifs d'une économie emixte», le pourquoi du peu de changement dans Porganisation syndicale formelle en regard des exigences d'une économie nouvelle, etc ...

Voilà donc un traité qui est beaucoup plus qu'un livre d'histoire et qui attaque de front, au moyen de la comparaison systématique et de la discussion de nature générale, les grands problèmes du syndicalisme contemporain. C'est ld sa valeur. Nous croyons qu'une telle méthode de travail apporte une dimension nécessaire à l'étude des institutions sociales, dimension que l'histoire seule ou l'analyse pure ne peuvent nous donner. A lire par tous ceux qui s'intéressent de près aux relations du travail.

Jean-Real Cardin

Chômage et évolution des structures, Bureau International du Travail, Genève, 1962, 245 pp.

Dans un pays où le chômage est aussi élevé que le Canada cette étude ne manque pas d'un certain intérêt. $\mathrm{Ce}$ pendant le changement de structures dont il est question ne semble pas de même nature que celui dont les canadiens sont présentement les témoins. Il s'agit de changement dans les structures de production dans une conjoncture où les emplois globaux sont suffisamment nombreux pour permettre aux 
travailleurs déplacés de se caser ailleurs après une période de réadaptation plutôt brève. Il ne s'agit pas d'un problème de changement dans les structures de production qui irait de pair avec un processus général de substitution de capital au travail. Le problème est plutốt celui d'une allocation différente du travail et non pas d'un remplacement du facteur travail par d'autres facteurs de production. Pour cette raison, l'intérêt immédiat de l'étude pour le règlement des problèmes canadiens de l'emploi est moindre que ne le laisse entendre le titre.

Il reste cependant que les problèmes traités sont universels même s'ils ne se manifestent pas avec la même acuité partout ou même si, ailleurs, d'autres types de chômage sont plus importants. L'étude est partagée en deux: la première partie précise la nature des changements de structures dont il est question. La seconde suggère les mesures qui tendent à faciliter les ajustements. La prudence avec laquelle on a rédigé le titre de la seconde partie est significative: il ne s'agit pas de panacée. Il ne s'agit pas de mesures qui règleraient nécessairement les problèmes. Il s'agit de mesures qui tentent - non pas de les régler - mais d'en faciliter le règlement. Cela, à mon avis, souligne bien les difficultés énormes posées par la solution des problèmes soulevés à l'occasion d'un changement dans les structures. Ils sont complexes, mouvants et fuyants. Les gouvernements les plus honnêtes sont parfois dans l'impossibilité de les régler à la satisfaction, non pas de tous, mais du plus grand nombre.

L'étude n'envisage donc le problème du chômage devant l'évolution des structures que dans une certaine conjoncture et pour un certain type d'évolution. De plus, l'analyse se situe surtout au niveau de la micro-économique c'est-à-dire au niveau de l'entreprise, ou de l'industrie, même si on suggère des remèdes que l'Etat seul peut imposer.

Ces remarques ne sont pas faites dans le but de minimiser les qualités de l'étude puisqu'elle est excellente. Elles ne sont là que pour signaler les limites à l'intérieur desquelles elle est vraiment excellente.
Wage Problems in Japan, Ministry of Foreign Affairs and Ministry of Labor, Tokyo, Japan, 1962, 26 pp.

Sans fouiller à fond les problèmes de la rémunération des travailleurs au Japon, cette publication en présente avec beaucoup de clarté les caractéristiques principales. On y signale avec à-propos dans quelle mesure certaines exigences sociales conditionnent la façon dont fonctionne le marché du travail. Par exemple, dans la détermination des salaires, on accorde plus d'importance qu'ici à l'âge et aux années de service. Ce sont là des exigences imposées par la société et c'est à l'intérieur de ces unités que s'applique la théorie de la productivité marginale. A cause de ces exigences, la distribution des emplois se fait d'une façon passablement différente de la nôtre. D'ailleurs, même chez nous, l'importance relative de l'ancienneté comme critère de détermination des salaires prend une importance croissante. C'est là un phénomène que le processus d'automation tend à accélérer. Malgré l'introduction dans les conventions collectives de clauses d'ancienneté de plus en plus strictes, il ne semble pas cependant que cette dernière ait chez nous un rôle aussi déterminant qu'au Japon.

En plus de souligner certaines caractéristiques du salariat, la publication tente de corriger la réputation que l'on fait au Japon en disant que cest un pays où les salaires sont bas. C'est là un argument souventes fois utilisé par les producteurs étrangers afin d'inciter leurs propres pays à protéger par des tarifs ou des quotas le marché local devant el'invasion» des exportations japonaises. La publication signale que les salaires versés aux travailleurs des industries d'exportation majeures se comparent avantageusement à ceux versés aux travailleurs européens des mêmes industries. Ce serait le cas entr'autres de l'industrie de l'acier et celle de la construction navale. Dans le cas de l'industrie textile, et celle des jouets, les salaires plus bas s'expliqueraient par le fait que les travailleurs sont surtout des jeunes femmes et que les bénéfices marginaux y sont - comme partout ailleurs au Japon - relativement plus importants qu'en Amérique. 